\title{
TRANSPLANTATION OF THE EN BLOC VASCULAR SYSTEM FOR CORONARY REVASCULARIZATION
}

\author{
Akio Matsuura, MD \\ Kenzo Yasuura, MD \\ Katsuhiko Yoshida, MD \\ Hideki Oshima, MD \\ Shiro Tomari, MD \\ Hideki Ishida, MD \\ Satsuki Mori, MD \\ Genyo Fujii, MD \\ Kazuki Iwata, MD
}

For related editorial, see p. 431.
Objectives: Use of the free gastroepiploic artery graft for coronary revascularization has not been very popular because of its inclination toward vasospasm. We hypothesized that the cause of free gastroepiploic artery spasm was the graft damage caused by an interruption of venous drainage from the graft. To solve this problem, we developed a new method of free gastroepiploic artery grafting.

Methods: From January 1997 to October 1999, 33 patients underwent coronary artery bypass grafting with the free gastroepiploic artery according to our new method. The gastroepiploic artery graft was harvested en bloc with its satellite veins. The gastroepiploic vein was anastomosed to the right atrial appendage for venous drainage simultaneously with the gastroepiploic artery being grafted in the aortocoronary position.

Results: A total of 96 distal anastomoses were performed, including 33 free gastroepiploic artery grafts according to our method, 33 in situ left internal thoracic artery grafts, 26 saphenous vein grafts, and 4 radial artery grafts. Neither operative nor hospital death occurred. Early postoperative angiography revealed that all of the 33 free gastroepiploic artery grafts performed with our method were patent without spasm, and flow competition occurred only in 2 of those grafts. On late angiography, all 15 free gastroepiploic artery grafts were patent without spasm.

Conclusions: The free gastroepiploic artery grafting with venous drainage technique we developed can prevent graft spasm, leading to improved patency rate. (J Thorac Cardiovasc Surg 2001;121:520-5)
$B^{2}$ ecause of their excellent long-term patency, arterial grafts are currently the first choice for coronary artery bypass grafting $(\mathrm{CABG})$. In recent years, the gastroepiploic artery (GEA) has been clinically used as a second reliable arterial material in addition to the internal thoracic artery (ITA). ${ }^{1,2}$ However, the use of in situ GEAs for CABG often shows flow competition between the graft and the native coronary artery when bypassed to the coronary artery with marginal stenosis in addition to showing a limited flow capacity. ${ }^{3-5}$ Moreover, the GEA pedicle can be damaged during future abdominal procedure. These disadvantages arise from its use as an in situ graft. Although removing the GEA and using it as a free graft in the aortocoronary position may resolve these problems, some reports warn of the risk of free GEA spasm and a low patency

From the Division of Cardiovascular Surgery, Cardiovascular Center, Aichi Prefectural Owari Hospital, Ichinomiya, Japan.

Copyright () 2001 by The American Association for Thoracic Surgery

$0022-5223 / 2001 \$ 35.00+0 \quad \mathbf{1 2 / 1 / 1 1 2 6 2 4}$

doi:10.1067/mtc.2001.112624 rate. $^{2,6}$ Accordingly, use of the free GEA graft has not been generally accepted. We hypothesized that the main cause of free GEA spasm was the graft damage caused by an interruption of venous drainage from the graft. To overcome this problem, we anastomosed the accompanied gastroepiploic vein (GEV) to the right atrial appendage simultaneously with the GEA grafting in the aortocoronary position. The purpose of this report is to present our experience in 33 patients to whom our new method of using a free GEA was applied and to assess the rationale of our hypothesis.

\section{Patients and methods}

Patient population. From January 1997 to October 1999, 33 patients underwent $\mathrm{CABG}$ performed by a single surgeon (A.M.) using the free GEA with venous drainage. There were 29 male patients and 4 female patients, with an average age of 58.2 years (range, 37-74 years). All patients signed informed consent forms before the operation. Preoperative clinical characteristics of the 33 patients are shown in Table I. One patient had a previous cholecystectomy. In 2 patients the free GEA grafting was aborted because of inadequate conduit size during the study period. 


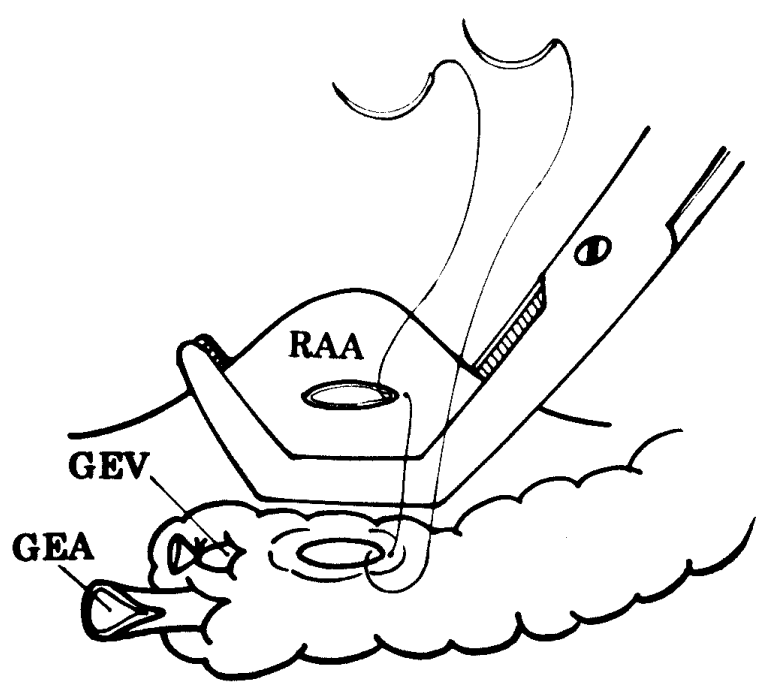

Fig 1. Technique for GEV-right atrial appendage (RAA) anastomosis. After initiation of cardiopulmonary bypass, GEV-right atrial appendage anastomosis is performed with ease.

Surgical technique. The median sternotomy was performed, and the incision in the midline abdominal fascia was extended to the midpoint between the xiphoid process and the umbilicus. The right GEA and vein graft was dissected along the distal half of the greater curvature of the stomach to the pylorus by means of an ultrasonic scissors (Harmonic Scalpel, Ultracision: Ethicon Endo-Surgery, Inc, Cincinnati, Ohio) to divide the branches to the stomach and omentum. After systemic heparinization, both proximal and distal ends of the graft were cut to be harvested. The distal end of the GEA and the proximal end of the GEV were cannulated with the soft, plastic, olive-tipped needles. A solution of blood and papaverine $(40 \mathrm{mg} / \mathrm{L})$ was injected gently into the vessels to dilate the GEA and GEV. Simultaneously, bleeding from the side branches was detected, and the GEV was identified and marked with a skin marker. A 7- to 8-mm long incision was made on the marked spot of the GEV $3 \mathrm{~cm}$ from the proximal end of the graft for anastomosis to the right atrial appendage. Both ends of the GEA were trimmed for anastomoses, and both ends of the GEV were ligated with sutures. Then the graft was placed in chilled Ringer's lactate solution until transplantation.

After initiation of the cardiopulmonary bypass with bicaval canulation, a 7- to 8-mm long incision was made on the right atrial appendage by using a side-bite clamp where the GEV was side-to-side anastomosed by means of a running suture with 2 threads of 7-0 polypropylene (Fig 1). Then the aorta was crossclamped, and cardioplegia was used to establish cardiac arrest. A 4-mm hole was made on the aorta on which the proximal end of the GEA was directly anastomosed with a 6-0 polypropylene suture. The distal anastomosis was also performed by means of a running 7-0 polypropylene suture.

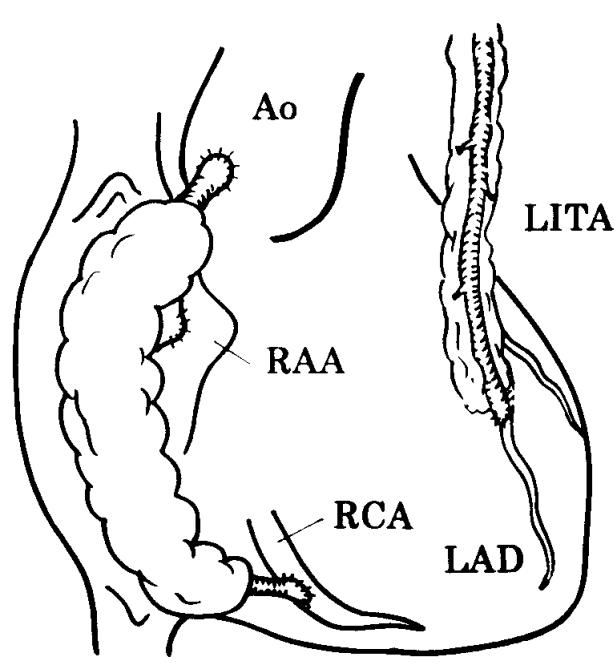

Fig 2. Schema of our technique. Proximal anastomosis of GEA with aorta, distal anastomosis of GEA to right coronary artery $(R C A)$, and GEV drainage into the right atrial appendage (RAA) are illustrated. Ao, Aorta; LITA, left ITA; $L A D$, left anterior descending artery.

Then other grafts were anastomosed during a single crossclamping (Fig 2). Fig 3 shows the difference between our technique and the conventional technique of free GEA grafting.

Angiographic evaluation. Follow-up angiography was performed 2 to 4 weeks after the operations in 33 patients. The results of the angiography were evaluated by 2 cardiologists in terms of patency, spasm, and string sign. The flow characteristics of the grafts were divided into 4 categories, according to the method described by Uchida and Kawaue ${ }^{4}$ : graft dependent, balanced, native dependent, and occluded. The cardiac catheterization was repeatedly performed 11 to 13 months postoperatively in 15 patients.

\section{Results}

A total of 96 distal anastomoses were performed (average, 2.9 per patient), including 33 free GEA grafts, 33 in situ left ITA grafts, 26 vein grafts, and 4 radial artery grafts. Free GEA grafting of our technique was applied to the main right coronary artery in 18 patients, the distal right coronary artery branches (posterior descending or posterolateral branches) in 13 patients, and the circumflex branches in 2 patients.

Neither operative nor hospital death was documented. Eighteen $(54.5 \%)$ of 33 patients were operated on without allogeneic blood transfusion. The results of early postoperative angiography are shown in Table II.

All of the 33 free GEA grafts were patent and with- 
Table I. Preoperative clinical characteristics

\begin{tabular}{lrc}
\hline Patient characteristics & No. & $\%$ \\
\hline Sex & & \\
$\quad$ Male & 29 & 87.9 \\
$\quad$ Female & 4 & 12.1 \\
Coronary disease & & \\
$\quad$ Double-vessel disease & 8 & 24.2 \\
$\quad$ Triple-vessel disease & 25 & 75.8 \\
Diabetes mellitus & 14 & 42.4 \\
Hypertension & 18 & 54.5 \\
Hypercholesterolemia & 15 & 45.5 \\
Total & 33 & \\
\hline
\end{tabular}

Table II. Early patency of the grafts

\begin{tabular}{|c|c|c|c|}
\hline & Patent & $\begin{array}{c}\text { Flow } \\
\text { competition }\end{array}$ & Occluded \\
\hline Free GEA & 31 & 2 & 0 \\
\hline Left ITA & 31 & 1 & 1 \\
\hline SVG & 25 & 0 & 1 \\
\hline RA & 4 & 0 & 0 \\
\hline
\end{tabular}

$S V G$, saphenous vein grafts; $R A$, radial artery grafts.

out spasm. In 12 patients the free GEA was put on the proximal right coronary artery with moderate stenosis (50\%-75\%). In this group all grafts were patent, but flow competition occurred in 2 grafts. In those grafts with flow competition, proximal stenosis was just $50 \%$. The flow pattern of the free GEA grafts in this group is shown in Table III.

Close observation of the angiography revealed that the small side branches run into the surrounding tissue from the main GEA (Fig 4). In one case the grafted GEV was cannulated in a retrograde manner through the right atrial appendage, and contrast medium was infused into it. The infused contrast medium flew through the GEV into the right atrium (Fig 5).

The duration of the follow-up was 59.9 patient years. One patient had ileus 6 months after the operation, which healed with conservative treatment. One patient died 30 months after the operation as a result of gastric cancer. Late angiographic control was obtained after a mean follow-up of 12 months in 15 patients. All of the 15 free GEA grafts were patent and without spasm, but the flow competition was repeatedly observed in 2 grafts without progression of the proximal stenosis.

\section{Discussion}

Lytle, ${ }^{7}$ Tanimoto, ${ }^{8}$ and their colleagues reported the use of the right GEA as a free graft. Advantages of a free GEA method are as follows: (1) the flow through a free GEA is larger than that through an in situ GEA;
Table III. Flow pattern of the free GEA grafts bypassed to the right coronary artery with moderate stenosis

\begin{tabular}{lcccc}
\hline $\begin{array}{l}\text { No. of } \\
\text { grafts }\end{array}$ & $\begin{array}{c}\text { Graft } \\
\text { dependent }\end{array}$ & Balanced & $\begin{array}{c}\text { Native } \\
\text { dependent }\end{array}$ & Occluded \\
\hline 12 & 9 & 1 & 2 & 0 \\
\hline
\end{tabular}

(2) freed grafts gain additional length to reach a distal coronary branch; and (3) graft damage with a future abdominal procedure can be avoided. However, some reports revealed that the free GEA grafts were prone to vasospasm, and the early patency rate of the free GEA grafts was lower than that of in situ grafts. ${ }^{2,6}$ At present, it is more common to use an in situ graft for the GEA than a free graft.

Foster and $\mathrm{Kranc}^{9}$ provided an explanation for the free GEA spasm. The GEA has well-developed vasa vasorum in its adventitial layers that penetrate deep into the media. When it is used as a free graft, the vasa vasorum of the vessel is disrupted at both ends, which leads to ischemia of the vessel wall and ultimately leads to graft spasm and occlusion. This explanation, however, is not accurate. The vasa vasorum of the harvested GEA can be preserved if the graft is dissected en bloc, together with its pedicle, including the satellite veins and the surrounding adipose tissue.

We have had 3 patients who underwent CABG with the traditional use of a free GEA. Unfortunately, the result could not encourage the use of a free GEA as an alternative arterial graft to the ITA. In 1 case the sternum closure was disturbed by the swollen periarterial tissues, mainly caused by blood congestion. In another case troublesome bleeding occurred when the graft was unclamped, and control of bleeding jeopardized the graft. In these 2 cases postoperative angiography revealed the grafts to be occluded. These distinct observations in traditional use of a free GEA indicated the necessity for another technique of free GEA grafting.

The major complications with a free GEA were swelling of the graft and bleeding from it, which were caused by a lack of venous drainage. We tested the harvested grafts before transplantation in 10 cases in this study. Heparinized blood was injected into GEA in the graft at a high pressure $(100 \mathrm{~mm} \mathrm{Hg})$ to detect bleeding from the side branches, and simultaneously, pressure change in the GEV was recorded. In all 10 grafts, venous pressure increased from $0 \mathrm{~mm} \mathrm{Hg}$ to over 40 $\mathrm{mm} \mathrm{Hg}$ within 2 minutes, and swelling of the grafts was observed. Then blood flow from the open end of the GEV was measured, which indicated a rate of 2.0 to $9.5 \mathrm{~mL} / \mathrm{min}$. The results of this clinical study suggest 

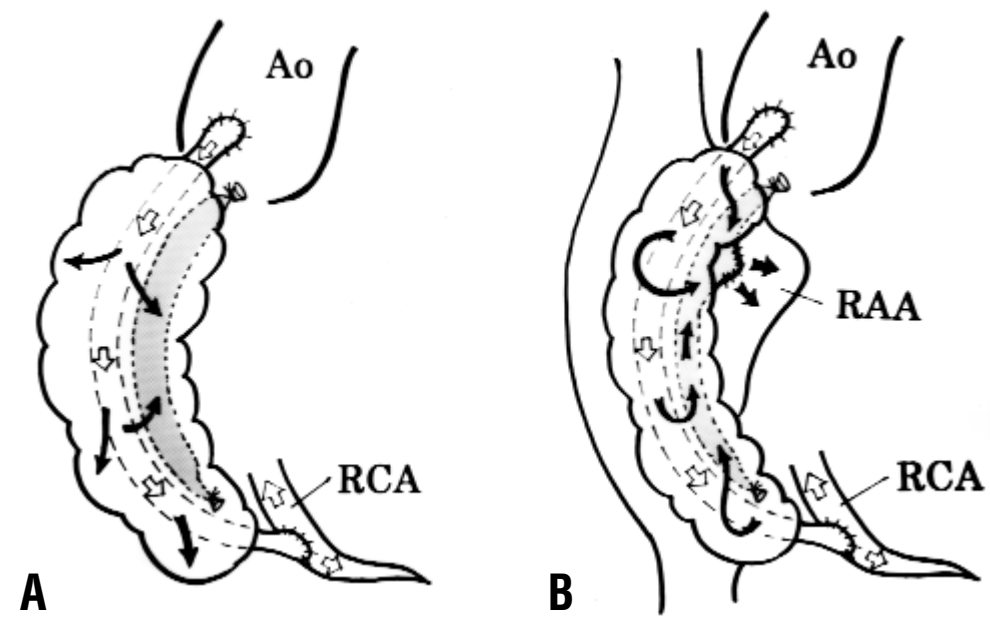

Fig 3. Schematic representation of the difference between our technique and the conventional technique of free GEA grafting. A, The graft is swollen with blood because of an interruption of venous drainage. B, The venous blood can drain through the GEV into the right atrium. White arrows indicate the arterial blood flow. Black arrows indicate the venous blood flow of the graft. $A o$, Aorta; $R C A$, right coronary artery; $R A A$, right atrial appendage.

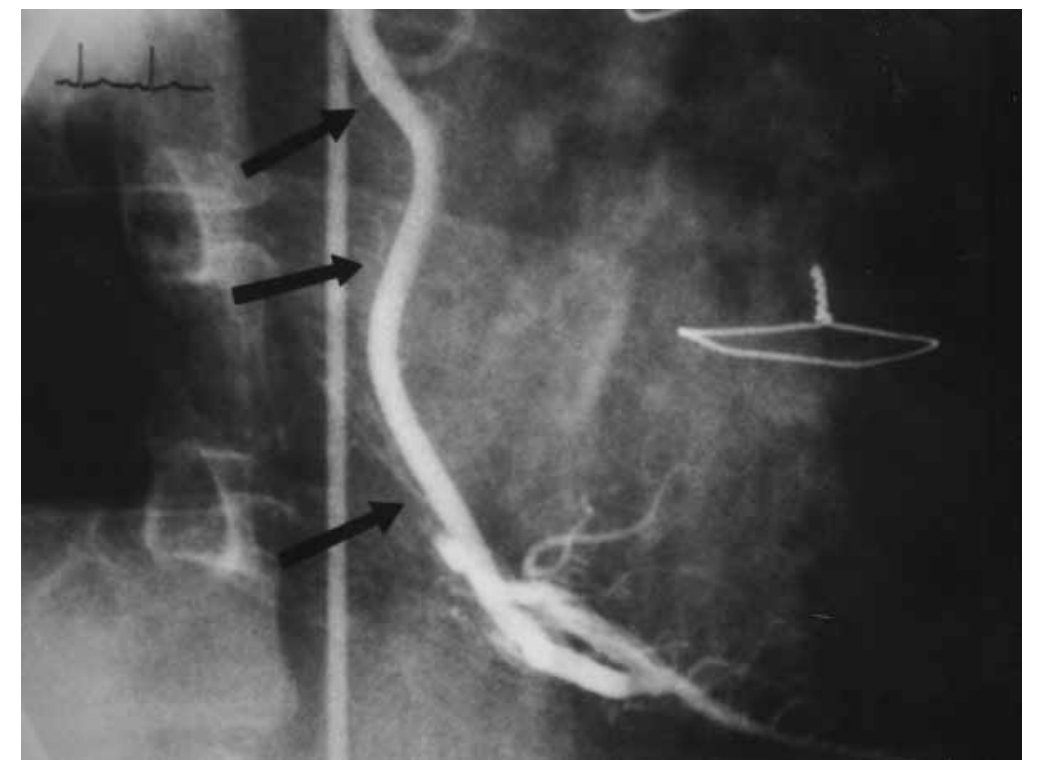

Fig 4. Postoperative angiogram of free GEA-right coronary artery anastomosis. Arrows indicate the small side branches running into the surrounding tissue.

that free GEA grafting in the aortocoronary position without venous drainage undoubtedly causes expansion of the GEV and swelling of the graft pedicle.

We hypothesized that the cause of the free GEA spasm might be graft damage caused by an interruption of venous drainage from the graft instead of the disruption of the vasa vasorum. To solve this problem, we anastomosed the accompanied GEV to the right atrial appendage for venous drainage simultaneously with the GEA grafting.
Because omentum is a living tissue that contains a lot of lymphatic, venous, and arterial vessels, free GEA grafting without venous drainage causes graft swelling. Mills and Everson ${ }^{6}$ reported edema in the pedicle when the GEV was ligated during harvest of the GEA graft. On the other hand, free ITA and other arterial grafts other than GEA grafts rarely include edema in the pedicle. This is the reason why only free GEA grafting requires venous drainage. Gagliardotto and colleagues ${ }^{10}$ reported a skeletonized in situ GEA tech- 


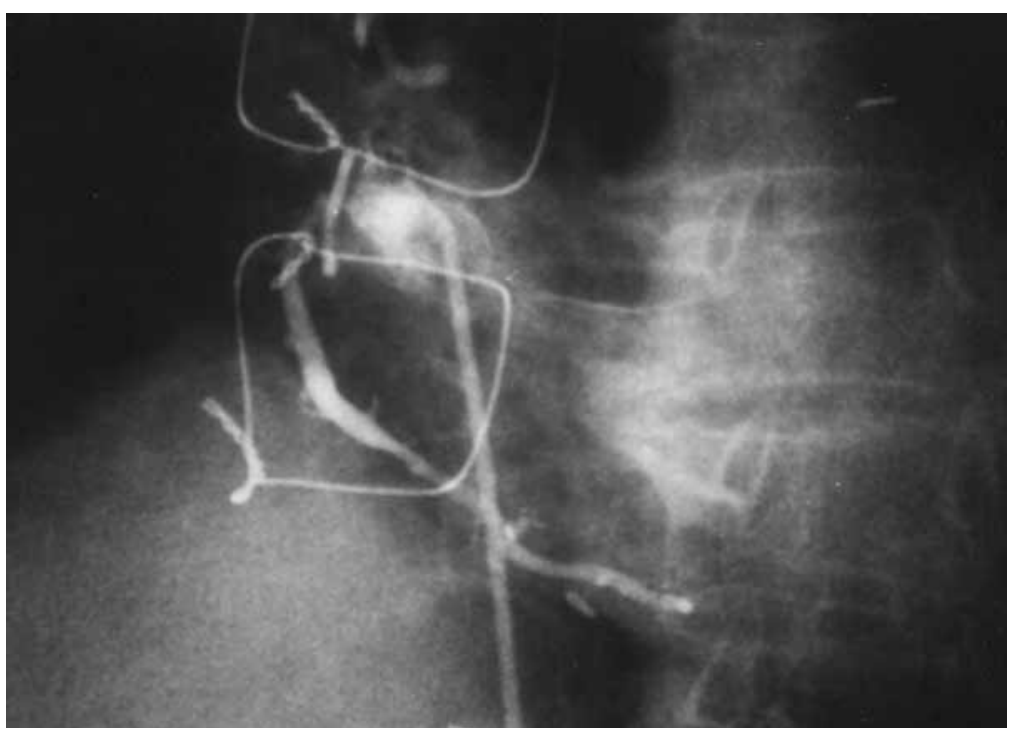

Fig 5. Postoperative angiogram of functioning venous system of the transplanted graft.

nique. A skeletonization technique may allow the prevention of free GEA swelling, but it is time consuming and technically demanding.

With our newly developed technique, the anastomosis of the GEV to the right atrial appendage can be done with a beating heart with the aid of cardiopulmonary bypass. As a result, the cardiac ischemic time is not prolonged. It takes less than 15 minutes to harvest free GEA grafts with the use of a Harmonic Scalpel.

Because the proximal end of the GEA is greater than $3 \mathrm{~mm}$ in diameter, the anastomosis can be constructed directly into the aorta with a continuous 6-0 polypropylene technique.

Bleeding from the side branches or swelling of the graft did not actually occur after unclamping in our experience.

Postoperative angiographic findings, in which the small side branches ran into the surrounding tissue from the main GEA with a patent grafted GEV, suggested the continued life of the transplanted grafts.

Suma and colleagues ${ }^{2}$ reported the lower patency rate of free GEA grafts (75\%) compared with that of in situ GEA grafts (95\%). Mills and Everson ${ }^{6}$ reported that 3 of 10 free GEA grafts had major vasospasm at early postoperative cardiac catheterization. In our study all of the 33 free GEA grafts with venous drainage showed a $100 \%$ patency rate without spasm. With regard to higher patency and immunity from vasospasm, our new technique of free GEA grafting with venous drainage is superior to the simple free GEA method.

Uchida and Kawaue ${ }^{4}$ warned of the risk of flow competition when an in situ GEA is used to bypass a moderate stenosis lesion. They reported that the grafts with a GEAdependent flow pattern were only $32.4 \%$ (11/34) in 34 in situ GEA grafts bypassed to coronary arteries with $75 \%$ stenosis. However, in our study 12 free GEA grafts were targeted to the moderate stenosis lesion at the proximal or mid right coronary artery (Table III). Under the same condition, a free GEA-dependent pattern was recognized in $75 \%(9 / 12)$ of cases. Our clinical experience demonstrated that the free GEA can be broadly used to bypass the coronary arteries with marginal stenosis. When GEA is used as a free graft instead of an in situ graft, blood flow is supplied directly from the ascending aorta, and the graft is larger at its distal end, it may have greater flow capacity and less sensitivity to competition flow.

Early and midterm angiographic results of the free GEA grafts with venous drainage demonstrate excellent patency rates and flows. Long-term patency of this graft is unknown. However, our new method is transplantation of the living omentum graft. The free GEA graft in our method is not only an arterial conduit but also a part of the living organ. We are hoping for a good long-term patency rate of this graft.

In conclusion, free GEA grafting with a simultaneous GEV drainage can prevent GEA spasm, leading to improvement in the patency rate in addition to providing more flow than in situ GEA. We advocate here that it is time to revive free GEA grafting, which has been neglected, with the help of our new method.

Received for publication June 15, 2000; revisions requested Sept 29, 2000; revisions received Oct 27, 2000; accepted for publication Oct 30, 2000. 
Address for reprints: Akio Matsuura, MD, Division of Cardiovascular Surgery, Cardiovascular Center, Aichi Prefectural Owari Hospital, 2135 Kariyasuka, Yamato-cho, Ichinomiya, Aichi 491-0934, Japan.

\section{REFERENCES}

1. Bergsma TM, Grandjean JG, Voors AA, Boonstra PW, Den Heyer $\mathrm{P}$, Ebels T. Low recurrence of angina pectoris after coronary artery bypass graft surgery with bilateral internal thoracic and right gastroepiploic arteries. Circulation 1998;97:2402-5.

2. Suma H, Wanibuchi Y, Terada Y, Fukuda S, Takayama T, Furuta S. The right gastroepiploic artery graft. J Thorac Cardiovasc Surg 1993;105:615-23.

3. Nakao K, Kawaue Y. Effect of coronary revascularization with the right gastroepiploic artery. J Thorac Cardiovasc Surg 1993; 106:149-53.

4. Uchida N, Kawaue Y. Flow competition of the right gastroepi- ploic artery in coronary revascularization. Ann Thorac Surg 1996;62:1342-6.

5. Yasuura K, Takagi Y, Ohara Y, Takami Y, Matsuura A, Okamoto $\mathrm{H}$. Theoretical analysis of right gastroepiploic artery grafting to right coronary artery. Ann Thorac Surg 2000;69:728-31.

6. Mills NL, Everson CT. Right gastroepiploic artery: A third arterial conduit for coronary artery bypass. Ann Thorac Surg 1989;47:706-11.

7. Lytle BW, Cosgrove DM, Ratliff NB, Loop FD. Coronary artery bypass grafting with the gastroepiploic artery. J Thorac Cardiovasc Surg 1989;97:826-31.

8. Tanimoto Y, Matsuda Y, Masuda T, Sakata K, Kobayashi Y, Hayashi Y, et al. Multiple free (aorta-coronary) gastroepiploic artery grafting. Ann Thorac Surg 1990;49:479-80.

9. Foster ED, Kranc MAT. Alternative conduit for aortocoronary bypass grafting. Circulation 1989;79(Suppl I):I-34-9.

10. Gagliardotto P, Coste P, Lazreg M, Dor V. Skeletonized right gastroepiploic artery used for coronary artery bypass grafting. Ann Thorac Surg 1998;66:240-2.

\section{THE THORACIC SURGERY FOUNDATION FOR RESEARCH AND EDUCATION}

\section{1-2002 Research Award Applications}

Information and applications for 2001-2002 Thoracic Surgery Foundation Research Grants, Research Fellowships, and Career Development Awards will be available beginning August 1, 2001. To request an application, please contact Lainie Castle at 312-644-6610 (telephone); 312-527-6635 (fax); or lcastle@sba.com (E-mail).

\section{TSFRE Donor Recognition Luncheon, May 2001}

The Thoracic Surgery Foundation is pleased to announce that its third annual Donor Recognition Luncheon will be held at noon on Tuesday, May 8, 2001, at The American Association for Thoracic Surgery's 81st Annual Meeting in San Diego, California. The Foundation will honor its New Century Society, Lifetime Members, and major individual and corporate contributors. 